\title{
Seletividade de herbicidas aplicados em pré e pós-emergência da cultura do cártamo
}

\author{
Selectivity of herbicides applied in pre and post emergence \\ of safflower culture
}

\begin{abstract}
Edicarlos Batista de Castro ${ }^{1 *}$, Diego Belapart ${ }^{2}$, Caio Antonio Carbonari ${ }^{2}$, Maurício Dutra Zanotto ${ }^{2}$, Ronei Ben², Gabrielle de Castro Macedo², Plinio Saulo Simões ${ }^{2}$
\end{abstract}

Resumo - O cultivo do cártamo tem aumentado nos últimos anos, principalmente para a produção de biodiesel. $\mathrm{O}$ controle de plantas daninhas a fim de evitar perdas na produtividade da cultura faz se necessário. Desta forma, uma alternativa que surge é o uso de herbicidas seletivos para a cultura. Objetivou-se verificar a seletividade de herbicidas em pré e pós-emergência para a cultura do cártamo. Foram conduzidos dois experimentos adotando-se delineamento em blocos casualizados com quatro repetições. Para o primeiro experimento com aplicações em pré-emergência os tratamentos foram arranjados em esquema fatorial $6 \times 2+1$, enquanto que para o segundo experimento, com as aplicações em pós-emergência os tratamentos foram dispostos em esquema fatorial 7 x $2+1$. Em ambos experimentos, o fator $\mathrm{A}$ correspondeu aos diferentes herbicidas e o fator $\mathrm{B}$ às doses utilizadas, e o tratamento adicional consistiu de testemunha sem aplicação de herbicidas. Foram realizadas avaliações visuais de fitointoxicação nas plantas aos 7, 14 e 21 dias após a aplicação, altura de plantas, estande e produtividade. Os herbicidas chlorimuron-ethyl (20 e $40 \mathrm{~g}$ i.a. ha ${ }^{-1}$ ), flumioxazin (50 e 100 g i.a. ha-1), imazaquin (161 e 322 g i.a. ha-1), s-metolachlor (1440 e $24000 \mathrm{~g}^{-1 . a . ~ h a-1}$ ) e sulfentrazone (300 g i.a. ha ${ }^{-1}$ ), aplicados em pré-emergência, e os herbicidas sethoxydim (184 g i.a. ha $^{-1}$ ), fluazifop-p-butyl (375 e $750 \mathrm{~g}^{\text {i.a. }} \mathrm{ha}^{-1}$ ) haloxyfop-methyl (60 e $120 \mathrm{~g}$ i.a. ha-1) e clethodim (108 g i.a. ha $\left.{ }^{-1}\right)$, aplicados em pós-emergência foram seletivos para a cultura do cártamo.

Palavras-chave: Carthamus tinctorius, controle químico, plantas daninhas

Abstract - The cultivation of safflower has increased in recent years, mainly for the production of biodiesel. However, it is necessary to weed control in order to avoid losses in productivity. In this way, an alternative that comes up is the use of selective herbicides for culture. This study aimed to verify the selectivity of different herbicides in pre and post-emergence to the safflower crop. Two experiments were conducted adopting a randomized block design with four replicates. For the first experiment with pre-emergence applications the treatments were arranged in a $6 \times 2+1$ factorial scheme, whereas for the second experiment with the post-emergence applications the treatments were arranged in a factorial scheme $7 \times 2+1$. For both experiments, the factor A corresponded to the different herbicides and factor $\mathrm{B}$ at the doses used and the additional treatment consisted of control without application

Recebido: Janeiro 20, 2017. Aceito: Outubro 16, 2017.

${ }^{1}$ Faculdade de Ciências Agronômicas Fazenda Lageado - FCA, Universidade Estadual Paulista "Júlio de Mesquita Filho" - UNESP, Rua José Barbosa de Barros, 1780, CEP 18610-307, Botucatu, SP, Brasil.

E-mail: castroeb@hotmail.com

${ }^{2}$ Universidade Estadual Paulista "Júlio de Mesquita Filho" - UNESP, Botucatu, SP, Brasil.

E-mail: diegobelapartt@hotmail.com; carbonari@fca.unesp.br; zanotto@fca.unesp.br; roneiben@hotmail.com; gabriellecmacedo@gmail.com; pliniosaulosimoes@hotmail.com 
of herbicides. Visual evaluations of intoxication were carried out in the plants at 7, 14 and 21 days after application (DAA), plant height, stand and productivity. The application of chlorimuron-ethyl (20 e 40 g i.a. ha ${ }^{-1}$ ), flumioxazin (50 e 100 g i.a. ha ${ }^{-1}$ ), imazaquin (161 e 322 g i.a. ha ${ }^{-1}$ ), s-metolachlor (1440 e $24000 \mathrm{~g}$ i.a. ha $\mathrm{h}^{-1}$ ) and sulfentrazone (300 g i.a. ha-1) in pre-emergence, and sethoxydim (184 g i.a. ha ${ }^{-1}$ ), fluazifop-p-butyl (375 e 750 g i.a. ha ${ }^{-1}$ ) haloxyfop-methyl (60 e 120 g i.a. ha-1) e clethodim (108 g i.a. ha $\left.{ }^{-1}\right)$ in post-emergence were selective for safflower.

Keywords: Carthamus tinctorius, chemical control, weed plants

\section{Introdução}

O cártamo (Carthamus tinctorius L.) é uma espécie oleaginosa da família Asteraceae, originária do Mediterrâneo (Ashri e Knowles, 1960). É uma cultura que apresenta o ciclo relativamente curto, em torno de 140 dias para as cultivares mais precoces, e pode ser cultivado na safrinha, na sequência de cultivos como a soja e o milho (Silva, 2013).

Nos últimos cinquenta anos houve um aumento significativo no seu cultivo, impulsionado pela produção de biodiesel que usa como matéria prima sues grãos, que tem alto teor de óleo, cerca de 40\% (Rech, 2012). No ano de 2016 registrou produção mundial de 647.374 toneladas de grãos (FAOSTAT, 2013).

O cártamo exibe grande capacidade de adaptação às mais variadas condições ecológicas predominantes nas regiões semiáridas, desenvolvendo-se bem em diferentes tipos de solos, e condições de altas temperaturas, baixa umidade do ar e déficit hídrico (Kizil et al., 2008; Bagheri e Sam-Dailiri, 2011; Bonamigo et al., 2013).

A produtividade do cártamo pode ser influenciada por diversos fatores. Dentre estes, destaca-se a interferência de plantas daninhas que pode causar, além da perda de produtividade, menor qualidade do produto e aumento do custo de produção da cultura (Ciuberkis et al., 2007). Para o controle das plantas daninhas, o uso de herbicidas surge como uma alternativa importante.

Apesar de ser uma cultura que vem ganhando espaço no País, existem poucos estudos referentes a seletividade de herbicidas para esta espécie. $\mathrm{Na}$ cultura do cártamo os herbicidas mais utilizados em outros países são os inibidores de EPSPs, ACCase e inibidores de divisão celular (Coronado, 2010). Entretanto se faz necessário conhecer a seletividade desses herbicidas, haja vista que, não existe nenhum herbicida no Brasil registrado para a cultura.

Para se determinar a seletividade de herbicidas a uma cultura, não basta apenas verificar os sintomas visuais de intoxicação, pois são conhecidos exemplos de herbicidas que reduziram a produtividade das culturas sem causar efeitos de intoxicação visual; há também exemplos de herbicidas que provocam injúrias acentuadas, mas que não interferem na produtividade da cultura (Velini et al., 2000; Negrisoli et al., 2004).

Desta forma, o objetivo do presente trabalho foi avaliar a seletividade de herbicidas aplicados em pré e pós-emergência da cultura do cártamo.

\section{Material e métodos}

Os experimentos foram conduzidos a campo nos meses de março a agosto de 2014. Aárea está situada nas coordenadas de Latitude $22^{\circ} 50$ ' 39,23" S e Longitude $48^{\circ} 25^{\prime} 28,53^{\prime \prime} \mathrm{O}$, a $780 \mathrm{~m}$ de altitude.

O clima da região, segundo a classificação de Koppen, se caracteriza como tropical, com inverno seco e verão chuvoso e quente. Os dados climáticos registrados durante o período de condução dos experimentos estão apresentados na Figura 1.

Foram realizadas coletas de solo em diferentes pontos da área experimental em profundidade de 0 a $20 \mathrm{~cm}$, para análise química e física do solo. O solo apresentava as seguintes caracteristicas: 568, 249 e $183 \mathrm{~g} \mathrm{dm}^{-3}$ de argila, silte e areia, respectivamente (textura argilosa), 


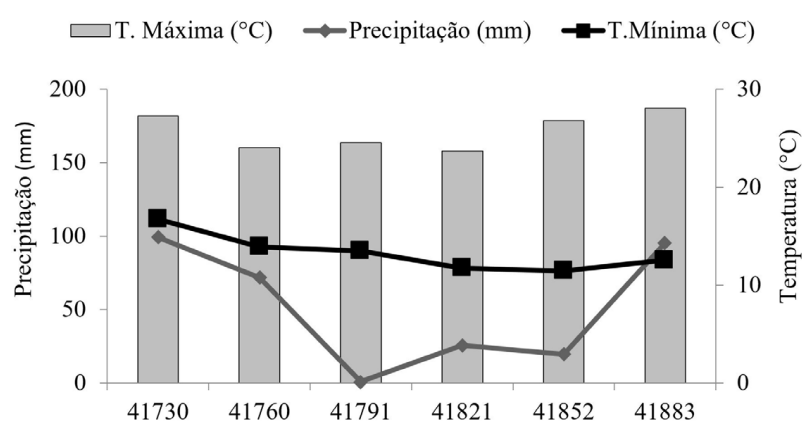

Figura 1. Precipitação pluvial, temperaturas máximas e mínimas registradas na área experimental durante o período de março a setembro de 2014.

$\mathrm{pH}\left(\mathrm{CaCl}_{2}\right)=4,7, \mathrm{M} . \mathrm{O} .=20 \mathrm{~g} \mathrm{dm}^{-3}, \mathrm{P}($ resina $)=7$ $\mathrm{mg} \mathrm{dm}{ }^{-3}, \mathrm{Al}^{3+}=1 \mathrm{mmolc} \mathrm{dm}^{-3}, \mathrm{H}+\mathrm{Al}=46 \mathrm{mmolc}$ $\mathrm{dm}^{-3}, \mathrm{~K}+=4$ mmolc $\mathrm{dm}^{-3}, \mathrm{Ca}^{2+}=24$ mmolc $\mathrm{dm}^{-}$ ${ }^{3}, \mathrm{Mg}^{2+}=13$ mmolc dm${ }^{-3}, \mathrm{SB}=41 \mathrm{mmolc} \mathrm{dm}^{-3}$; CTC (T) $=87$ mmolc dm $^{-3}$ e V\% $=47$.

Foram conduzidos dois experimentos adotando-se o delineamento em blocos casualizados com quatro repetições. Para o primeiro experimento, com aplicações em pré- emergência, os tratamentos foram arranjados em esquema fatorial $6 \times 2+1$; enquanto que para o segundo experimento, com as aplicações em pós-emergência, os tratamentos foram arranjados em esquema fatorial $7 \times 2+1$. Em ambos experimentos, o Fator A correspondeu aos herbicidas e $\mathrm{o}$ Fator $\mathrm{B}$ às doses utilizadas, $\mathrm{o}$ tratamento adicional foi composto por testemunha sem aplicação de herbicidas, sendo todas as parcelas capinadas ao longo do ciclo da cultura, a fim de eliminar o efeito da interferência das plantas daninhas sobre a produtividade do cártamo. Na Tabela 1 estão apresentados a relação de herbicidas e as respectivas doses utilizadas em cada experimento.

O genótipo de cártamo utilizado na semeadura do experimento foi o IMA 2237. Na semeadura, realizada em abril de 2014, foram distribuídas 20 sementes por metro linear, as quais foram depositadas em profundidade de aproximadamente $3 \mathrm{~cm}$. Cada parcela foi constituída de cinco linhas com $5 \mathrm{~m}$ de comprimento, as quais se apresentavam espaçadas a $0,45 \mathrm{~m}$ entre si (área bruta $=11,25 \mathrm{~m}^{2}$ ). Apenas as três linhas centrais foram avaliadas, sendo descartados também como bordadura $0,5 \mathrm{~m}$ de cada extremidade da parcela, totalizando área útil de $5,4 \mathrm{~m}^{2}$.

As aplicações foram realizadas com um pulverizador costal de pressão constante a base de $\mathrm{CO}_{2}$, pressão de $196,1 \mathrm{kPa}$, equipado com cinco bicos XR 110.02, espaçados em 0,5 m, com volume de calda aplicado equivalente a $200 \mathrm{~L} \mathrm{ha}^{-1}$. Todas as aplicações foram realizadas respeitando o mínimo de $50 \%$ de umidade e máximo de $30^{\circ} \mathrm{C}$ de temperatura. A aplicação dos herbicidas em pré-emergência ocorreu no dia seguinte a semeadura do cártamo e a aplicação em pós-emergência foi realizada aos 28 dias após a emergência (DAE) da cultura. No momento da aplicação em pós-emergência, as plantas de cártamo se encontravam em estádio vegetativo, apresentando aproximadamente 8 folhas verdadeiras e $15 \mathrm{~cm}$ de altura.

No experimento com herbicidas aplicados em pré-emergência, foram realizadas avaliações visuais de fitointoxicação aos 7, 14 e 21 DAE. Para o experimento com herbicidas aplicados em pós-emergência, as avaliações foram realizadas aos 7, 14, 21 dias após a aplicação (DAA). Foi utilizada a escala percentual de notas, na qual " 0 " corresponde a ausência de injúrias visíveis e " 100 " à morte das plantas, conforme descrito pela SBCPD (1995).

Na ocasião da colheita dos experimentos, em setembro de 2014, foi determinado o estande de plantas de cártamo, altura das plantas e produtividade. O estande de plantas foi determinado pela contagem de plantas presentes em dois metros lineares. A altura de plantas de cártamo foi mensurada pela medição da distância referente a base da planta no solo até a última folha expandida, medindo 10 plantas por parcela. A produtividade de grãos foi determinada pela colheita manual das plantas, que foram trilhadas e, posteriormente, 
Tabela 1. Herbicidas utilizados na cultura do cártamo.

\begin{tabular}{|c|c|c|c|c|c|}
\hline Herbicidas & Nome comercial & $\begin{array}{c}\text { Doses } \\
\left(\mathrm{g} \text { i.a. } \text { ha }^{-1}\right)\end{array}$ & $\begin{array}{l}\text { Mecanismo } \\
\text { de Ação }\end{array}$ & $\begin{array}{c}\text { Formulação/ } \\
\text { Fornecedor }\end{array}$ & Experimento \\
\hline Chlorimuron-ethyl & Classic & 20 e 40 & ALS & WG/DUPONT & PRÉ \\
\hline Flumioxazin & Flumyzin 500 & 50 e 100 & Protox & WP/SUMITOMO & PRÉ \\
\hline Imazaquin & Topgan & 161 e 322 & ALS & SL/MILÊNIA & PRÉ \\
\hline Metribuzin & Sencor 480 & 240 e 480 & Fotossistema II & SC/BAYER & PRÉ \\
\hline S-metolachlor & Dual Gold & 1440 e 2880 & $\begin{array}{l}\text { Inibidor da } \\
\text { Parte Aérea }\end{array}$ & EC/SYNGENTA & PRÉ \\
\hline Sulfentrazone & Boral $500 \mathrm{SC}$ & 300 e 600 & Protox & $\mathrm{SC} / \mathrm{FMC}$ & PRÉ \\
\hline Sethoxydim & Poast & 184 e 368 & ACCase & DC/BASF & PÓS \\
\hline Lactofen & Cobra & 90 e 180 & Protox & CE/BAYER & PÓS \\
\hline Imazethapyr & Pivot 100 SL & 50 e 100 & ALS & SL/BASF & PÓS \\
\hline Fluazifop-p-butyl & Fusilade $250 \mathrm{EW}$ & 375 e 750 & ACCase & EW/SYNGENTA & PÓS \\
\hline Haloxyfop-methyl & Verdict R & 60 e 120 & ACCase & CE/SYNGNETA & PÓS \\
\hline Bentazon & Basagran 600 & 450 e 900 & Fotossistema II & $\mathrm{SL} / \mathrm{BASF}$ & PÓS \\
\hline Clethodim & $\begin{array}{c}\text { Select } 240 \\
\text { EC + lanzer(adj) }\end{array}$ & 108 e 216 & ACCase & EC/ARYSTA & PÓS \\
\hline
\end{tabular}

determinou-se a massa dos grãos, com correção da umidade para $13 \%$.

Os dados foram submetidos à análise de variância pelo teste $\mathrm{F}$ a 5\% de probabilidade, e as médias comparadas pelo teste de Tukey a $5 \%$ de probabilidade

\section{Resultados e discussão}

\section{Seletividade de herbicidas aplicados em pré-emergência na cultura do cártamo}

Apenas o herbicida s-metolachlor, para a menor dose utilizada (1440 g i.a. ha ${ }^{-1}$ ), não causou intoxicação visual às plantas de cártamo, independentemente da época de avaliação. É possível observar que, a aplicação deste herbicida na maior dose (2880 g i.a. ha $\left.{ }^{-1}\right)$, as plantas de cártamo passaram a apresentar sintomas de injúrias. A aplicação dos herbicidas flumioxazin e sulfentrazone, ambos nas menores doses avaliadas (50 g i.a. ha-1 e 300 g i.a. ha ${ }^{-1}$, respectivamente), resultou em fitointoxicação inferior ao metribuzin em todas as avaliações. De forma geral, as menores doses dos herbicidas testados foram àquelas que proporcionaram menores níveis de fitointoxicação (Tabela 2).
Estes resultados demonstram que a cultura do cártamo apresenta sensibilidade diferencial em função da dose utilizada. Assim como observado por Lamego et al. (2011) que, ao aplicarem diferentes doses de s-metolachlor, verificaram o aumento de fitointoxicação na cultura do feijão com o incremento da dose.

O cártamo apresentou diferentes médias de altura após a aplicação dos herbicidas em função do tratamento utilizado (Tabela 3). Os herbicidas imazaquin, sulfentrazone e chlorimuron-ethyl, aplicados em ambas as doses, bem como o metribuzin na dose de $240 \mathrm{~g}$ i.a. ha ${ }^{-1}$, proporcionaram maior restrição na taxa de crescimento das plantas, resultando em menores valores de altura. É possível observar que para a média dos herbicidas (Fator A) não houve diferença significativa. Já para as médias das doses (Fator B), verifica-se que imazaquin e sulfentrazone foram inferiores ao demais tratamentos em altura.

Para a variável estande de plantas, os herbicidas flumioxazin (50 g i.a. ha ${ }^{-1}$ ) e s-metolachlor (1440 g i.a. ha $\left.{ }^{-1}\right)$ foram semelhantes à testemunha (Tabela 3). A aplicação dos herbicidas chlorimuron (20 e 40 g i.a. ha $\left.{ }^{-1}\right)$, flumioxazin (100 g i.a. ha $\left.{ }^{-1}\right)$, imazaquin (161 e 322 g i.a. ha ${ }^{-1}$ ), metribuzin (240 e 480 g i.a. ha $\left.{ }^{-1}\right)$, s-metolachlor (2880 g i.a. ha $\left.{ }^{-1}\right)$ 
Tabela 2. Fitointoxicação (\%) da cultura do cártamo após a aplicação de herbicidas em préemergência.

\begin{tabular}{|c|c|c|c|c|c|c|c|c|c|}
\hline \multirow{2}{*}{ Tratamentos } & \multicolumn{3}{|c|}{7 DAA } & \multicolumn{3}{|c|}{14 DAA } & \multicolumn{3}{|c|}{21 DAA } \\
\hline & Dose 1 & Dose 2 & Média & Dose 1 & Dose 2 & Média & Dose 1 & Dose 2 & Média \\
\hline chl & $75 \mathrm{aA}$ & $74 \mathrm{aA}$ & $74 \mathrm{a}$ & $80 \mathrm{aA}$ & $83 \mathrm{aA}$ & $81 \mathrm{a}$ & $85 \mathrm{aA}$ & $85 \mathrm{aA}$ & $85 \mathrm{a}$ \\
\hline flu & $50 \mathrm{bB}$ & $85 \mathrm{aA}$ & $68 \mathrm{a}$ & $56 \mathrm{bB}$ & $91 \mathrm{aA}$ & $74 \mathrm{a}$ & $59 \mathrm{cB}$ & $92 \mathrm{aA}$ & $75 \mathrm{a}$ \\
\hline ima & $66 \mathrm{abB}$ & $81 \mathrm{aA}$ & $74 \mathrm{a}$ & $74 \mathrm{abB}$ & 89 aA & $81 \mathrm{a}$ & $84 \mathrm{abA}$ & $93 \mathrm{aA}$ & $88 \mathrm{a}$ \\
\hline met & $80 \mathrm{aA}$ & $65 \mathrm{abB}$ & $73 \mathrm{a}$ & $85 \mathrm{aA}$ & $76 \mathrm{aA}$ & $80 \mathrm{a}$ & $93 \mathrm{aA}$ & $83 \mathrm{abA}$ & $88 \mathrm{a}$ \\
\hline s-met & $0 \mathrm{cB}$ & $50 \mathrm{bA}$ & $25 \mathrm{~b}$ & $0 \mathrm{cB}$ & $55 \mathrm{bA}$ & $27 \mathrm{~b}$ & $0 \mathrm{~dB}$ & $63 \mathrm{bA}$ & $31 \mathrm{~b}$ \\
\hline sul & $49 \mathrm{bB}$ & $74 \mathrm{aA}$ & $61 \mathrm{a}$ & $56 \mathrm{bB}$ & $83 \mathrm{aA}$ & $69 \mathrm{a}$ & $64 \mathrm{bcB}$ & $93 \mathrm{aA}$ & $78 \mathrm{a}$ \\
\hline Média & $53 \mathrm{~b}$ & $71 \mathrm{a}$ & & $59 \mathrm{~b}$ & $79 \mathrm{a}$ & & $64 \mathrm{~b}$ & $85 \mathrm{a}$ & \\
\hline CV (\%) & \multicolumn{2}{|c|}{15,9} & & \multicolumn{2}{|c|}{14,4} & \multicolumn{4}{|c|}{12,9} \\
\hline DMS Herbicida & \multicolumn{2}{|c|}{21,2} & & \multicolumn{2}{|c|}{21,2} & & \multicolumn{2}{|c|}{20,6} & \\
\hline DMS Dose & \multicolumn{2}{|c|}{14,3} & & \multicolumn{2}{|c|}{14,3} & & \multicolumn{2}{|c|}{13,9} & \\
\hline
\end{tabular}

chl $=$ chlorimuron-ethyl; flu = flumioxazin; ima $=$ imazaquin; met $=$ metribuzin; s-met $=$ S-metolachlor; sul = sulfentrazone. Médias seguidas da mesma letra minúscula na coluna e maiúscula na linha não diferem entre si pelo teste de Tukey $(\mathrm{p} \geq 0,05)$.

Tabela 3. Altura e estande de plantas de cártamo após a aplicação em pré-emergência.

\begin{tabular}{|c|c|c|c|c|c|c|c|c|c|}
\hline \multirow{2}{*}{ Tratamentos } & \multicolumn{3}{|c|}{ Estande (plantas $\left.\mathbf{m}^{-1}\right)$} & \multicolumn{3}{|c|}{ Altura $(\mathrm{cm})$} & \multicolumn{3}{|c|}{ Produtividade $\left(\mathrm{kg} \mathrm{ha}^{-1}\right)$} \\
\hline & Dose 1 & Dose 2 & Média & Dose 1 & Dose 2 & Média & Dose 1 & Dose 2 & Média \\
\hline chl & $7,7 \mathrm{aA}$ & $6,3 \mathrm{aA}$ & $6,9 \mathrm{a}$ & (*) $80 \mathrm{abA}$ & $73 \mathrm{abA}$ & $77 \mathrm{abc}$ & (*) $706 \mathrm{aA}$ & (*) $659 \mathrm{aA}$ & $682 \mathrm{a}$ \\
\hline flu & (*) $9,2 \mathrm{aA}$ & $6,9 \mathrm{aA}$ & $8,0 \mathrm{a}$ & (*) $87 \mathrm{aA}$ & (*) $82 \mathrm{aA}$ & $85 \mathrm{a}$ & (*) $802 \mathrm{aA}$ & (*) $695 \mathrm{aA}$ & 749 a \\
\hline ima & $6,2 \mathrm{aA}$ & $6,3 \mathrm{aA}$ & $6,2 \mathrm{a}$ & $73 \mathrm{bA}$ & $63 \mathrm{bB}$ & $68 \mathrm{c}$ & (*) $603 \mathrm{aA}$ & (*) $541 \mathrm{aA}$ & $572 \mathrm{a}$ \\
\hline met & 7,6 aA & $5,7 \mathrm{aA}$ & $6,6 \mathrm{a}$ & $75 \mathrm{abA}$ & (*) $82 \mathrm{aA}$ & $78 \mathrm{ab}$ & $519 a$ & $439 \mathrm{aA}$ & 479 a \\
\hline s-met & (*) $9,7 \mathrm{aA}$ & $6,7 \mathrm{aA}$ & $8,2 \mathrm{a}$ & (*) $84 \mathrm{abA}$ & (*) $77 \mathrm{aA}$ & $81 \mathrm{ab}$ & (*) $682 \mathrm{aA}$ & (*) $567 \mathrm{aA}$ & $625 \mathrm{a}$ \\
\hline sul & $6,9 \mathrm{aA}$ & $4,3 \mathrm{aA}$ & $5,6 \mathrm{a}$ & $71 \mathrm{bA}$ & $74 \mathrm{abA}$ & $73 \mathrm{bc}$ & (*) $668 \mathrm{aA}$ & $467 \mathrm{aA}$ & $567 \mathrm{a}$ \\
\hline Média & $7,9 \mathrm{a}$ & $6,0 \mathrm{~b}$ & & $78 \mathrm{a}$ & $75 \mathrm{a}$ & & $663 \mathrm{a}$ & $561,5 \mathrm{a}$ & \\
\hline Testemunha & \multicolumn{2}{|c|}{$13,2 *$} & & \multicolumn{2}{|c|}{$90,4^{*}$} & & \multicolumn{2}{|c|}{$966,5 *$} & \\
\hline CV $(\%)$ & \multicolumn{2}{|c|}{32,5} & & \multicolumn{2}{|c|}{8,17} & & \multicolumn{2}{|c|}{32,6} & \\
\hline $\begin{array}{c}\text { DMS } \\
\text { Herbicida }\end{array}$ & \multicolumn{2}{|c|}{5,13} & & \multicolumn{2}{|c|}{13,5} & & \multicolumn{2}{|c|}{443,7} & \\
\hline DMS Dose & \multicolumn{2}{|c|}{3,5} & & \multicolumn{2}{|c|}{9,1} & & \multicolumn{2}{|c|}{299,4} & \\
\hline
\end{tabular}

chl = chlorimuron-ethyl; flu = flumioxazin; ima = imazaquin; met $=$ metribuzin; s-met $=$ S-metolachlor; sul = sulfentrazone. Médias seguidas da mesma letra minúscula na coluna e maiúscula na linha não diferem entre si pelo teste de Tukey $(\mathrm{p} \geq 0,05)$. Médias representadas por “*” não diferem da testemunha sem herbicida, segundo o Teste de Dunnett $(\mathrm{p} \geq 0,05)$.

e sulfentrazone (300 e 600 g i.a. ha ${ }^{-1}$ ) causaram redução no estande da cultura, porém não diferiram entre si quanto ao nível de fitointoxicação causado.

A produtividade da cultura foi reduzida apenas com a aplicação dos herbicidas metribuzin (240 e 480 g i.a. ha-1) e sulfentrazone (600 g i.a. ha-1) quando comparados à testemunha sem aplicação. Isso demonstra que nem todos os herbicidas que causaram sintomas de fitointoxicação nas plantas de cártamo reduziram a produtividade da cultura
(Tabela 3). De acordo com Biffe et al. (2010), a seletividade de um herbicida não pode ser baseada apenas pelos sintomas de fitointoxicação. Segundo Melhorança (2000), alguns herbicidas podem reduzir a produtividade das culturas sem causar efeitos visualmente detectáveis de intoxicação, e outros podem provocar injúrias tóxicas evidentes na cultura e não causar redução na produtividade.

Os sintomas de fitointoxicação, altura e estande após a aplicação em pré-emergência dos herbicidas 
chlorimuron (20 e $40 \mathrm{~g}$ i.a. ha-1), flumioxazin (50 e 100 g i.a. ha-1), imazaquin (161 e 322 g i.a. ha ${ }^{-1}$ ), s-metolachlor ( 1440 e $2880 \mathrm{~g}$ i.a. ha $\left.{ }^{-1}\right)$ e sulfentrazone (300 g i.a. ha-1) não resultaram em redução da produtividade da cultura (Tabela 3 ).

\section{Seletividade de herbicidas aplicados em pós-emergência na cultura do cártamo}

Os herbicidas lactofen e bentazon, aos 7 DAA, foram responsáveis pelos maiores percentuais de fitointoxicação do cártamo quando aplicados em pós-emergência da cultura. Aos 14 e 21 DAA, lactofen, bentazon e imazethapyr causaram os maiores danos à cultura. (Tabela 4).

Os herbicidas inibidores da ACCase (sethoxydim, fluazifop-p-butyl, haloxyfop-methyl e clethodim) não causaram fitointoxicação quando aplicados em pós-emergência do cártamo. Tal resultado pode ser explicado pelo fato destes herbicidas serem empregados para o controle de plantas daninhas monocotiledôneas, sendo utilizados, portanto, de forma seletiva em culturas dicotiledôneas (Jasper et al., 2015). Segundo Barroso et al. (2010), o mecanismo que inibe a ACCase, bloqueia a síntese de lipídeos apenas nas plantas susceptíveis, impossibilitando a formação da membrana celular e causando desestruturação dos tecidos em formação.

A fitointoxicação ocasionada na cultura do cártamo após a aplicação em pós-emergência está relacionada com o mecanismo de ação dos herbicidas, pois, lactofen, imazethapyr e bentazon são herbicidas com espectro de ação sobre dicotiledôneas. Os resultados do presente trabalho estão de acordo com Reis et al. (2014), que observaram fitointoxicação após a aplicação em pós-emergência do imazethapyr na cultura do girassol. Inoue et al. (2014), ao aplicar o herbicida bentazon, também observaram severas injúrias para a cultura do pinhão manso.

As maiores produtividades do cártamo foram observadas com a aplicação dos herbicidas fluazifop (375 e 750 g i.a. ha ${ }^{-1}$ ), haloxyfop (60 e 120 g i.a. ha-1), sethoxydim (184 g i.a. ha $\left.{ }^{-1}\right)$ e clethodim (108 gi.a. ha-1) (Tabela 5). As menores doses dos herbicidas proporcionaram maior produtividade média da cultura. A altura e estande das plantas foram reduzidas apenas pela aplicação de bentazon (450 e 900 g i.a. ha ${ }^{-1}$ ).

Tabela 4. Fitointoxicação (\%) da cultura do cártamo após a aplicação de herbicidas em pósemergência.

\begin{tabular}{|c|c|c|c|c|c|c|c|c|c|}
\hline \multirow{2}{*}{ Tratamentos } & \multicolumn{3}{|c|}{7 DAA } & \multicolumn{3}{|c|}{14 DAA } & \multicolumn{3}{|c|}{21 DAA } \\
\hline & Dose 1 & Dose 2 & Média & Dose 1 & Dose 2 & Média & Dose 1 & Dose 2 & Média \\
\hline set & $0 \mathrm{cA}$ & $0 \mathrm{cA}$ & $0 \mathrm{c}$ & $0 \mathrm{cA}$ & $0 \mathrm{bA}$ & 0 & $0 \mathrm{cA}$ & $0 \mathrm{cA}$ & $0 \mathrm{c}$ \\
\hline lac & $80 \mathrm{aA}$ & $86 \mathrm{aA}$ & $83 \mathrm{a}$ & $78 \mathrm{bB}$ & $84 \mathrm{aA}$ & $81 \mathrm{~b}$ & $81 \mathrm{bA}$ & $81 \mathrm{bA}$ & $81 \mathrm{~b}$ \\
\hline ima & $46 \mathrm{bA}$ & $54 \mathrm{bA}$ & $50 \mathrm{~b}$ & $84 \mathrm{aA}$ & $88 \mathrm{aA}$ & $86 \mathrm{a}$ & $87 \mathrm{abA}$ & $85 \mathrm{abA}$ & $86 \mathrm{ab}$ \\
\hline flu & $0 \mathrm{cA}$ & $0 \mathrm{cA}$ & $0 \mathrm{c}$ & $0 \mathrm{cA}$ & $0 \mathrm{bA}$ & $0 \mathrm{c}$ & $0 \mathrm{cA}$ & $0 \mathrm{cA}$ & $0 \mathrm{c}$ \\
\hline hal & $0 \mathrm{cA}$ & $0 \mathrm{cA}$ & $0 \mathrm{c}$ & $0 \mathrm{cA}$ & $0 \mathrm{bA}$ & $0 \mathrm{c}$ & $0 \mathrm{cA}$ & $0 \mathrm{cA}$ & $0 \mathrm{c}$ \\
\hline ben & $84 \mathrm{aA}$ & $85 \mathrm{aA}$ & $84 a$ & $87 \mathrm{aA}$ & $88 \mathrm{aA}$ & $87 \mathrm{a}$ & $90 \mathrm{aA}$ & $93 \mathrm{aA}$ & $91 \mathrm{a}$ \\
\hline cle & $0 \mathrm{cA}$ & $0 \mathrm{cA}$ & $0 \mathrm{c}$ & $0 \mathrm{cA}$ & $0 \mathrm{bA}$ & $0 \mathrm{c}$ & $0 \mathrm{cA}$ & $0 \mathrm{cA}$ & $0 \mathrm{c}$ \\
\hline Média & $30 \mathrm{a}$ & $32 \mathrm{a}$ & & $35 \mathrm{a}$ & $37 \mathrm{~b}$ & & $36,8 \mathrm{a}$ & $37,0 \mathrm{a}$ & \\
\hline CV (\%) & \multicolumn{2}{|c|}{17,4} & & \multicolumn{2}{|c|}{8,6} & \multicolumn{4}{|c|}{10,7} \\
\hline DMS Herbicida & \multicolumn{2}{|c|}{$8,4,0$} & & \multicolumn{2}{|c|}{4,5} & \multicolumn{4}{|c|}{6,1} \\
\hline DMS Dose & \multicolumn{2}{|c|}{2,9} & & \multicolumn{2}{|c|}{1,6} & \multicolumn{4}{|c|}{2,1} \\
\hline
\end{tabular}

set = sethoxydim; lac = lactofen; ima = imazethapyr; flu = fluazifop-p-butyl; hal = haloxyfop-methyl; ben = bentazon; cle $=$ clethodim. Médias seguidas da mesma letra, minúscula na coluna não diferem significativamente pelo teste de Tukey $(\mathrm{p} \geq 0,05)$. 
Tabela 5. Altura e estande de plantas de cártamo após a aplicação em pós-emergência.

\begin{tabular}{|c|c|c|c|c|c|c|c|c|c|}
\hline \multirow{2}{*}{ Tratamentos } & \multicolumn{3}{|c|}{ Estande (plantas $\left.\mathbf{m}^{-1}\right)$} & \multicolumn{3}{|c|}{ Altura $(\mathrm{cm})$} & \multicolumn{3}{|c|}{ Produtividade $\left(\mathrm{kg} \mathrm{ha}^{-1}\right)$} \\
\hline & Dose 1 & Dose 2 & Média & Dose 1 & Dose 2 & Média & Dose 1 & Dose 2 & Média \\
\hline set & $(*) 11,8 \mathrm{aA}$ & $(*) 10,8 \mathrm{aA}$ & $11,25 \mathrm{ab}$ & (*) $90 \mathrm{aA}$ & (*) $90 \mathrm{aA}$ & $90 \mathrm{ab}$ & (*) $966 \mathrm{aA}$ & $621 \mathrm{abB}$ & $794 \mathrm{a}$ \\
\hline lac & (*) $9,4 \mathrm{aA}$ & $\left.{ }^{*}\right) 10,4 \mathrm{aA}$ & $9,9 \mathrm{~b}$ & (*) $80 \mathrm{aA}$ & (*) $80 \mathrm{aA}$ & $80 \mathrm{~b}$ & $334 \mathrm{bA}$ & $304 \mathrm{bA}$ & $319 b$ \\
\hline ima & $\left(^{*}\right) 9,9 \mathrm{aA}$ & (*) $9,6 \mathrm{aA}$ & $9,7 \mathrm{~b}$ & (*) $77 \mathrm{aA}$ & (*) $80 \mathrm{aA}$ & $79 \mathrm{~b}$ & $320 \mathrm{bA}$ & $301 \mathrm{bA}$ & $310 \mathrm{~b}$ \\
\hline flu & $\left(^{*}\right) 13,6 \mathrm{aA}$ & $(*) 13,4 \mathrm{aA}$ & $13,5 \mathrm{a}$ & $(*) 87 \mathrm{aA}$ & (*) $89 \mathrm{aA}$ & $88 \mathrm{ab}$ & (*) $869 \mathrm{aA}$ & (*) $826 \mathrm{aA}$ & $848 \mathrm{a}$ \\
\hline hal & $\left(^{*}\right) 12,2 \mathrm{aA}$ & $\left(^{*}\right) 11,3 \mathrm{aA}$ & $11,8 \mathrm{ab}$ & (*) $93 \mathrm{aA}$ & (*) $93 \mathrm{aA}$ & 93 a & $\left(^{*}\right) 1072 \mathrm{aA}$ & 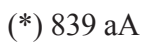 & $956 \mathrm{a}$ \\
\hline ben & $2,7 \mathrm{bA}$ & $2,3 \mathrm{bA}$ & $2,5 \mathrm{c}$ & $47 \mathrm{bA}$ & $41 \mathrm{bA}$ & $44 \mathrm{c}$ & $278 \mathrm{bA}$ & $204 \mathrm{bA}$ & $241 \mathrm{~b}$ \\
\hline cle & $\left(^{*}\right) 12,9 \mathrm{aA}$ & $\left(^{*}\right) 12,3 \mathrm{aA}$ & $12,6 \mathrm{ab}$ & $(*) 87 \mathrm{aA}$ & (*) $90 \mathrm{aA}$ & $88 \mathrm{ab}$ & $(*) 831 \mathrm{aA}$ & $605 \mathrm{abA}$ & $718 \mathrm{a}$ \\
\hline Média & 10,3 a & $10,0 \mathrm{a}$ & & 80 & 80 & & 667 a & $529 \mathrm{~b}$ & \\
\hline Testemunha & \multicolumn{3}{|c|}{$13,3^{*}$} & \multicolumn{2}{|c|}{$90^{*}$} & \multicolumn{4}{|c|}{$1069^{*}$} \\
\hline CV (\%) & \multicolumn{2}{|c|}{19,1} & & \multicolumn{2}{|c|}{9,5} & \multicolumn{4}{|c|}{33,3} \\
\hline $\begin{array}{c}\text { DMS } \\
\text { Herbicida }\end{array}$ & \multicolumn{2}{|c|}{4,4} & & \multicolumn{2}{|c|}{16,8} & \multicolumn{4}{|c|}{458,6} \\
\hline DMS Dose & \multicolumn{2}{|c|}{2,8} & & \multicolumn{2}{|c|}{10,9} & \multicolumn{4}{|c|}{299,1} \\
\hline
\end{tabular}

set = sethoxydim; lac = lactofen; ima = imazethapyr; flu = fluazifop-p-butyl; hal = haloxyfop-methyl; ben = bentazon; cle $=$ clethodim. Médias seguidas da mesma letra minúscula na coluna e maiúscula na linha não diferem entre si pelo teste de Tukey $(\mathrm{p} \geq 0,05)$. Médias representadas por “*” não diferem da testemunha sem herbicida, segundo o Teste de Dunnett $(\mathrm{p} \geq 0,05)$.

Foi possível verificar que os herbicidas que causaram maior fitointoxicação foram também os que causaram decréscimo na produtividade. Sendo assim, fica evidente que o cártamo é sensível aos herbicidas latifolicidas aplicados em pós-emergência no presente estudo. Assim como Souza et al. (2014), ao avaliar a fitointoxicação e produtividade da cultura do crambe após a aplicação de lactofen e bentazon observaram a morte das plantas de crambe. Também verificou-se a redução do crescimento na cultura da mandioca com a aplicação de lactofen (Silva et al., 2012).

\section{Conclusões}

Os herbicidas chlorimuron-ethyl (20 e 40 g i.a. ha-1), flumioxazin (50 e 100 g i.a. ha${ }^{1}$ ), imazaquin (161 e $322 \mathrm{~g}$ i.a. ha ${ }^{-1}$ ), s-metolachlor (1440 e 24000 gi.a. ha-1 $)$ e sulfentrazone (300 g i.a. ha ${ }^{-1}$ ), aplicados em pré-emergência, e os herbicidas sethoxydim (184 gi.a. ha ${ }^{-1}$ ), fluazifop-p-butyl (375 e 750 g i.a. ha ${ }^{-1}$ ), haloxyfop-methyl (60 e $120 \mathrm{~g}$ i.a. ha ${ }^{-1}$ ) e clethodim (108 g i.a. ha ${ }^{-1}$ ), aplicados em pós-emergência foram seletivos para a cultura do cártamo.

\section{Referências}

Ashri, A.; Knowles, P.F. Cytogenetics of safflower (Carthamus L.) species and their hybrids. Agronomy Journal, v.52, n.1, p.11-17, 1960.

Bagheri, B.; Sam-Dailiri, M. Effect of water stress on agronomic traits of safflower spring (Carthamus tinctorius). Australian Journal of

Basic and Applied Sciences, v.5, n.12, p.26212624, 2011.

Barroso, A.L.L.; Dan, H.A.; Procópio, S.O.; Toledo, R.E.B.; Sandaniel, C.R.; Braz, G.B.P.; Cruvinel, K.L. Eficácia de herbicidas inibidores da ACCase no controle de gramíneas em lavouras de soja.

Planta Daninha, v.28, n.1, p.149-157, 2010.

Biffe, D.F.; Constantin, J.; Oliveira Junior, R.S.; Rios, F.A.; Franchini, L.H.M.; Gemelli, A.; et al. Avaliação de herbicidas para dois cultivares de mandioca. Planta Daninha, v.28, n.4, p.807$816,2010$.

Bonamigo, T.; Fortes, A.M.T.; Pinto, T.T.; Gomes, F.M.; Silva, J.; Baturi, C.V. Interferência alopática 
de folhas de cártamo sobre espécies oleaginosas. Biotemas, v.26, n.2, p.1-8, 2013.

Ciuberkis, S.; Bernotas, S.; Raudonius, S.; Felix, J. Effect of weed emergence time and intervals of weed and crop competition on potato yield. Weed Technology, v.21, n.3, p.213-218, 2007.

Coronado, L.M. El cultivo del cártamo (Carthamus tinctorius L.) en México. Ciudad Obregon-México: SGI, 2010, 96p.

FAOSTAT - Food And Agriculture Organization Of The United Nations. Crops. 2013. Disponível em: <http://faostat.fao.org/site/567/DesktopDefault. aspx? PageID=567\#ancor $>$. Acesso em: 14 out. 2016.

Inoue, M.H.; Pereira, K.M.; Mendes, K.F.; Santos, E.G.; Dallacort, R.; Possamai, A.C.S. Seletividade de herbicidas para pinhão manso em condições de casa de vegetação e campo. Bioscience Journal, v.30, n.2, p.791-801, 2014.

Jasper, S.P.; Velini, E.D.; Rossetto, M.R.M.; Carbonari, C.A.; Trindade, M.L.B. Aplicação em subdose do haloxyfop-methyl na aveia-preta. Ciência Rural, v.45, n.4, p.637-643, 2015.

Kizil, S.; Çakmak, O.; Kiric, S.; Ínam, M. Comprehensive study on Safflower (Carthamus tinctorius L.) in semi-arid conditions. Biotechnology, Biotechnological Equipment, v.4, n.1, p.947953, 2008.

Lamego, F.P.; Basso, C.J.; Vidal, R.A.; Trezzi, M.M.; Santi, A.L.; Ruchel, Q.; et al. Seletividade dos herbicidas s-metolachlor e alachlor para o feijão-carioca. Planta Daninha, v.29, n.4, p.877883, 2011.

Melhorança, A.L. Seletividade dos herbicidas diclosulam, flumetsulam e cloransulam em diversas cultivares de soja. Revista Brasileira de Herbicidas, v.1, n.2, p.119-122, 2000.

Negrisoli, E.; Velini, E.D.; Tofoli, G.R.; Cavenaghi, A.L.; Martins, D.; Morelli, J.L.; Costa, A.G.F.
Seletividade de herbicidas aplicados em préemergência na cultura de cana-de-açúcar tratada com nematicidas. Planta Daninha, v.22, n.4, p.567-575, 2004.

Rech, J. Desempenho agronômico do cártamo (Carthamus tinctorius L.) em função da época de semeadura e do controle químico da mancha de alternaria. 2012. 45 f. Dissertação (Mestrado em Agronomia/Produção Vegetal) - Universidade Federal da Grande Dourados, Dourados, 2012.

Reis, R.M.; Souza, M.F.; Queiroz, G.P.; Siebert, I.G.; Silva, D.; Ferreira, E.A.; Silva, A.A. Tolerância do girassol a herbicidas aplicados em pós-emergência. Revista Brasileira de Herbicidas, v.13, n.1, p.15-22, 2014.

Silva, C.J. Caracterização agronômica e divergência genética de acessos de cártamo. 2013. 51 f. Tese (Doutorado em Agronomia/ Agricultura) - Faculdade de Ciências Agronômicas, Universidade Estadual Paulista "Júlio de Mesquita Filho", Botucatu, 2013.

Silva, D.V.; Santos, J.B.; Carvalho, F.P.; Ferreira, E.A.; França, A.C.; Fernandes, J.S.C.; et al. Seletividade de herbicidas pós-emergentes na cultura da mandioca. Planta Daninha, v.30, n.4, p.835-841, 2012.

Sociedade Brasileira da Ciência das Plantas Daninhas. Procedimentos para instalação, avaliação e análise de experimentos com herbicidas. Londrina, 1995. 42 p.

Souza, G.S.F.; Vitorino, H.S.; Fioreze, A.C.C.L.; Pereira, M.R.R.; Martins, D. Seletividade de herbicidas na cultura de crambe. Semina: Ciências Agrárias, v.35, n.1, p.161-168, 2014.

Velini, E.D.; Martins, D.; Manoel, L.A.; Matsuoka, S.; Travain, J.C.; Carvalho, J.C. Avaliação da seletividade da mistura de oxyfluorfen e ametryne, aplicada em pré ou pós-emergência, a dez variedades de cana-de-açúcar (cana-planta). Planta Daninha, v.18, n.1, p.123-134, 2000. 